\title{
Investigation of a tunable and switchable multi-wavelength bidirectional Brillouin-erbium fiber ring laser
}

\author{
F. WANG ${ }^{1 *}$, R.Y. YUAN ${ }^{2}$ \\ ${ }^{1}$ School of Physical Science and Technology, Southwest University, \\ Chongqing 400715, China \\ ${ }^{2}$ Institute of Electrical and Electronic Engineering, Chongqing University of Technology, \\ Chongqing 400054, China
}

*Corresponding author: wangf17@swu.edu.cn

\begin{abstract}
A multi-wavelength bidirectional Brillouin-erbium fiber ring laser with switchable Brillouin frequency spacing (BFS) is proposed and experimentally demonstrated. In the presented Brillouin-erbium ring laser, including an optical amplifier and a highly nonlinear fiber, and without any optical isolator, due to Rayleigh scattering, stimulated Brillouin scattering, and cascaded four-wave mixing initiated successively by the Brillouin pump (BP) light, the odd- and even-order Stokes lines are generated and circulate in the opposite direction in the ring cavity. The BP light and Stokes-induced Rayleigh backscattering light also simultaneously circulate in the ring cavity. Only by adjusting BP power, the gain competition between Brillouin based Stokes and cavity modes' oscillation can be controlled, the laser output can be conveniently switched between single BFS and odd- or even-order double BFS. In addition, under the certain BP power conditions, the proposed multi-wavelength Brillouin-erbium fiber laser also can realize switchable odd- or even-order Stokes generation and Stokes generation with single BFS, with an increasing wavelength number in turn, only by simply adjusting pump power of the erbium-doped fiber amplifier. Stability and wavelength tunability of the proposed multi-wavelength bidirectional Brillouin-erbium fiber ring laser are also investigated, respectively.
\end{abstract}

Keywords: Rayleigh scattering, stimulated Brillouin scattering, four-wave mixing, lasers and laser optics.

\section{Introduction}

Multi-wavelength fiber lasers (MWFLs) have attracted extensive attention due to their potential applications in various fields, such as optical communications [1] , optical fiber sensors system [ㄹ] , microwave signal processing []ㅡ, and so on. In order to achieve multi -wavelength lasing, a variety of filters is incorporated into MWFL such as Fabry-Pérot filter []], fiber grating [ㄷ], Mach-Zehnder interferometers [ㅁ] , nonlinear optical loop 
mirror [7], Lyot-Sagnac filter [ $\underline{8}$ ], and so on. One of the viable approaches to achieve low-cost multi-wavelength lasing is utilizing stimulated Brillouin scattering (SBS) in the fiber. Multi-wavelength Brillouin fiber lasers (MBFLs) take advantage of Rayleigh backscattering from Brillouin gain medium itself, a Doppler frequency shift that is introduced when the Brillouin pump (BP) light is backscattered by a moving acoustic wave, which cause the frequency downshift of the Stokes light [9]. As a result of this feedback-seeding, multiple Brillouin-Stokes (BS) lines can be generated successfully. The amount of the Stokes frequency shift is determined by the speed of the acoustic wave in the fiber core, and it is typically around $10 \mathrm{GHz}$ for silica-based fibers.

Owing to several important advantages including free filter, stable output, low threshold power, narrow linewidth and equal wavelength spacing, MBFLs have been extensively investigated. For example, a broadband MBFL with an operating wavelength range of $1500-1600 \mathrm{~nm}$ and a frequency separation of $\sim 9.28 \mathrm{GHz}$ based on four -wave mixing (FWM) in a dual MBFL cavity was reported [10]. A simple way was proposed to generate a double-Brillouin-frequency spaced multi-wavelength. This structure utilizes the reflection property of an isolator (ISO) and fiber loop mirror to allow the light to be absorbed twice and thus only even Stokes waves in clockwise direction are generated [11]. A MBFL with triple Brillouin frequency spacing (BFS) by employing a modular structure was proposed and experimentally investigated [ 3$]$. However, the Brillouin frequency intervals generated by these methods are fixed and cannot be flexibly adjusted, they will be limited in many practical applications. Therefore, some methods for adjusting frequency spacing of multiple Stokes lights have been reported. For example, a hybrid Brillouin-thulium MWFL with switchable spacing, corresponding to the single and double BFS at $2 \mu \mathrm{m}$ Brillouin-thulium fiber laser cavities was reported in [12]. A novel tunable MBFL with switchable frequency spacing by using optical gain and absorption during the SBS process was reported [13]. A method for obtaining frequency-spaced switchable MWBFL corresponding to single, double, triple and quadruple Brillouin frequency shifting by cascading a multiple Brillouin gain fiber was demonstrated [14].

Due to weak distributed feedback, the number of generated Stokes lines in MBFLs is usually relatively limited. One solution is that another gain medium is considered to incorporate into laser cavity to compensate cavity loss and improve performance of MBFL. The combination of SBS and another gain mechanism have been realized in different hybrid cavity structures such as the multi-wavelength Brillouin-erbium fiber lasers (MBEFLs) [15-17], multi-wavelength Brillouin-thulium fiber lasers (MBTFLs) [18-20], multi-wavelength Brillouin-Raman fiber lasers (MBRFLs) [12, 21], and so on.

In this paper, we propose and experimentally demonstrate a simple method to generate multiple Stokes sequences with switchable frequency spacing. Multi-wavelength lasing with single BFS, odd-order or even-order double BFS can be conveniently achieved, only by adjusting BP power. When a continuous wave $(\mathrm{CW})$ as BP is injected into a bidirectional ring cavity including an optical amplifier and a highly nonlinear fiber (HNLF), Rayleigh scattering (RS), SBS and cascaded four-wave mixing (CFWM) effect are inspired by the BP light, and the gain competition between Brillouin based 
Stokes and cavity modes' oscillation are also stimulated successively. The odd- and even-order Stokes waves circulate in opposite directions within the ring cavity, meanwhile, the corresponding Rayleigh backscattering for these Stokes waves is also excited. When the BP power is sufficiently strong, the power of generated Stokes is relatively high, which makes the optical amplifier in the ring cavity being in deep saturation state, and these weak Rayleigh back light is suppressed. So, the odd- and even-order Stokes lines with double BFS are generated in the counterclockwise and clockwise direction of the ring cavity, respectively. When the BP power is reduced, the power of generated Stokes decreases, and an optical amplifier gradually recovers from a deep saturation state. When these weak scattering lights get enough gain and are amplified to be close to or equivalent to the power of the odd- and even-order Stokes in the optical amplifier, the Stokes lasing is switched from double BFS to single BFS. The influence of the BP power and pump power of erbium-doped fiber amplifier (EDFA) on Stokes generation from MBEFL is also investigated, respectively. In addition, under the certain BP power conditions, the proposed MBEFL also can realize switchable odd- or even-order Stokes generation or Stokes generation with single BFS, with an increasing wavelength number in turn, only by simply adjusting EDFA pump power. In the stability test for the system output, low fluctuation of less than $1.80 \mathrm{dBm}$ within 60 minutes demonstrates that the proposed MBEFL has a good stability. When the BP wavelength is tuned within a range of about $7 \mathrm{~nm}$ from 1557.075 to $1564.073 \mathrm{~nm}$, stable and clear MBEFL output still can be obtained, and we believe that the range of wavelength tuning can be increased by optimizing the system structure.

\section{Experimental setup and principle}

Experimental setup of the proposed MBEFL is illustrated in Fig. 1. A CW emitted from a tunable laser source (TLS), with a full width at half maximum (FWHM) linewidth of $1 \mathrm{MHz}$, through an ISO and polarization controller (PC1), is injected into the Brillouin -erbium ring cavity via $10 \%$ port of an optical coupler (OC1). ISO prevents optical feedback from entering the TLS. PC1 is used to adjust polarization state of the BP light

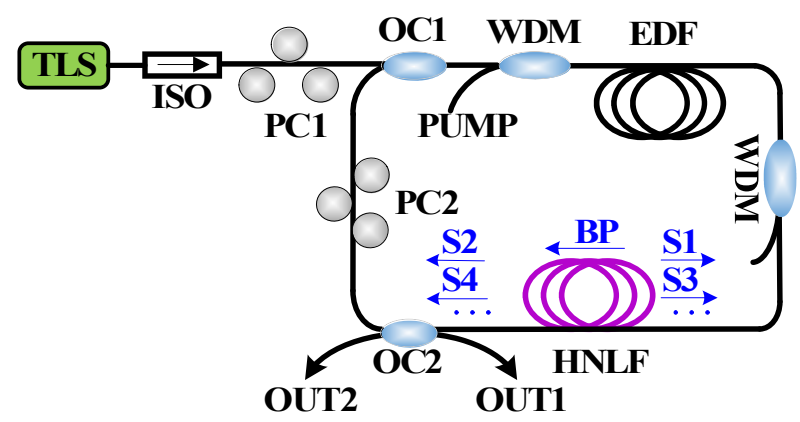

Fig. 1. Experimental setup of the proposed multi-wavelength Brillouin-erbium fiber laser with switchable frequency spacing, TLS: tunable laser source, ISO: isolator, PC: polarization controller, OC: optical coupler, WDM: wavelength division multiplexer, EDF: erbium-doped fiber, HNLF: high nonlinear fiber. 
sent to the ring cavity. A segment of 1-meter long erbium-doped fiber (EDF) with extremely high doping concentration (Liekki Er110, absorption of $110 \mathrm{~dB} / \mathrm{m}$ at $1530 \mathrm{~nm}$ ) and pumped by a $980 \mathrm{~nm}$ laser diode delivering maximum output of $700 \mathrm{~mW}$, with the help of a $980 / 1550 \mathrm{~nm}$ wavelength division multiplexer (WDM), provides a relatively high gain and self-lasing threshold. A segment of 1-km length HNLF with the nonlinear coefficient of $\sim 10 \mathrm{~W}^{-1} \mathrm{~km}^{-1}$, a zero-dispersion wavelength of $\sim 1550 \mathrm{~nm}$, and a dispersion slop of $0.03 \mathrm{ps} / \mathrm{nm}^{2} / \mathrm{km}$ serves as the Brillouin gain medium to induce RS, SBS and CFWM effect for creating a broadband multi-wavelength lasing. The ratio of the OC2 is $10: 90$ and its $10 \%$ ports are used as the output ports, and the remaining $90 \%$ of energy is kept in the ring cavity. The output spectrum was analyzed by using an optical spectrum analyzer (OSA, Anritsu MS9740A) with a minimum resolution of $0.03 \mathrm{~nm}$.

The proposed MBEFL is mainly based on RS, SBS and CFWM effect in the Brillouin -erbium ring cavity. When the BP light transmits forward in HNLF, RS random feedback is caused. SBS strengthens these feedbacks and stimulates FWM to expand the Stocks spectrum. During the SBS process, the interaction of the BP and Stokes wave in the HNLF can be described by the coupled intensity differential equation,

$$
\begin{aligned}
& \frac{\mathrm{d} I_{\mathrm{P}}}{\mathrm{d} z}=-\frac{g_{\mathrm{B}}}{A_{\text {eff }}} I_{\mathrm{P}} I_{\mathrm{S}}-\alpha I_{\mathrm{P}} \\
& \frac{\mathrm{d} I_{\mathrm{S}}}{\mathrm{d} I_{\mathrm{P}}}=-\frac{g_{\mathrm{B}}}{A_{\text {eff }}} I_{\mathrm{P}} I_{\mathrm{S}}+\alpha I_{\mathrm{S}}
\end{aligned}
$$

where $I_{\mathrm{P}}$ represents intensity of the $\mathrm{BP}, z$ is propagation axis along the fiber, $g_{\mathrm{B}}$ is Brillouin gain coefficient, $A_{\text {eff }}$ is effective core area of the HNLF, $I_{\mathrm{S}}$ represents intensity of Stocks waves and $\alpha$ is optical attenuation coefficient along the HNLF. By solving these coupled equations using the shooting method [22], it is found that intensity of the generated Stocks waves will grow exponentially from the far end to the near end of the HNLF as

$$
I_{\mathrm{S}}(0)=I_{\mathrm{S}}(L) \exp \left(\frac{g_{\mathrm{B}} P_{0} L_{\text {eff }}}{A_{\text {eff }}-\alpha L}\right)
$$

where $P_{0}=I_{\mathrm{P}}(0) A_{\text {eff }}$, and $P_{0}$ is input pump power, $L$ and $L_{\text {eff }}$ are length and effective length of the HNLF, respectively. In practice, $I_{\mathrm{S}}(0)$ and $I_{\mathrm{S}}(L)$ are established by Stocks photon from noise or spontaneous Brillouin scattering occurring throughout the fiber length. In general, SBS threshold is defined as the input pump power at which the backward scattered Stocks power becomes equal to the pump power at the fiber output [17], which can be expressed as

$$
P_{\text {th }}=\frac{21 A_{\mathrm{eff}} \alpha}{g_{\mathrm{B}}[1-\exp (-\alpha L)]}
$$



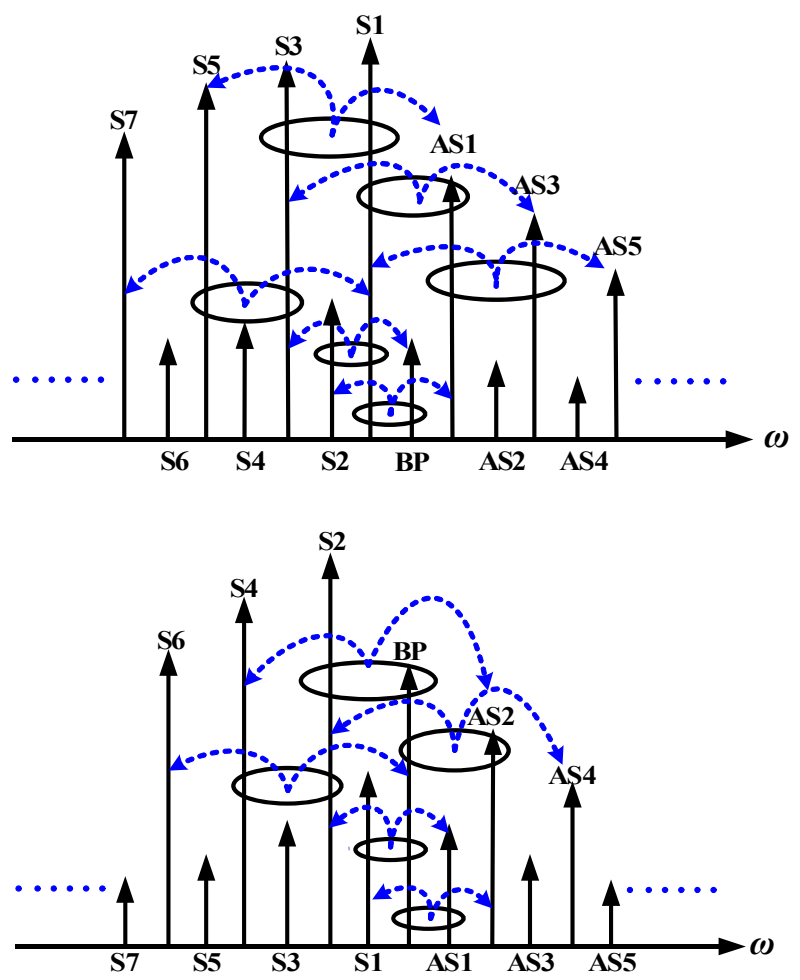

a

b

Fig. 2. The illustration of stimulated Brillouin scattering and multiple four-wave mixing processes within the Brillouin-erbium ring cavity observed from the counterclockwise direction (a) and from the clockwise direction (b).

When generated Stokes waves loop in the ring cavity, the effective length for interaction between the pump and Stocks waves is extended. According to Eq. (4), SBS threshold can be lowered, thus a higher SBS conversion efficiency is achieved.

The illustration of SBS and multiple FWM processes within the Brillouin-erbium ring cavity is shown in Fig. 2. The CW emitted from TLS is employed as BP and injected into the ring cavity through ISO, $\mathrm{PC} 1$ and $10 \%$ port of OC1; the BP power is amplified in EDFA, and then is launched into HNLF. When the BP power increases and SBS exceeds threshold power, the 1 st order Stokes (S1) with a frequency $\omega_{\mathrm{S} 1}$ is generated, which is downshifted by $\Delta \omega_{\mathrm{B}}$ from the BP frequency $\omega_{\mathrm{P}}$, namely, $\omega_{\mathrm{S} 1}=\omega_{\mathrm{P}}-\Delta \omega_{\mathrm{B}}$. $\mathrm{S} 1$ propagates along anti-clockwise direction within the ring cavity, opposite to the transmission direction of the BP. Then the power of S1 is also amplified in the EDFA before reaching the opposite end of the HNLF. Via OC2, the $90 \%$ energy of BP and $\mathrm{S} 1$ remain in the ring cavity, which drastically reduces the SBS threshold. The BP and S1 will experience pump depletion and strong Brillouin amplification, respectively. When S1 propagates in the HNLF, it acts as another BP and generates the second-order BS lines (S2) with a frequency $\omega_{\mathrm{S} 2}\left(\omega_{\mathrm{S} 2}=2 \omega_{\mathrm{S} 1}-\omega_{\mathrm{P}}=\omega_{\mathrm{P}}-2 \Delta \omega_{\mathrm{B}}\right)$ in the clockwise 
direction, meantime, and the first-order anti-Stokes signal (AS1) with a frequency $\omega_{\mathrm{AS} 1}$ $\left(\omega_{\mathrm{AS} 1}=2 \omega_{\mathrm{P}}-\omega_{\mathrm{S} 1}=\omega_{\mathrm{P}}+\Delta \omega_{\mathrm{B}}\right)$ is also generated by mixing between $\omega_{\mathrm{S} 1}$ and $\omega_{\mathrm{P}}$. With increasing of the BP power, the power of S1 and S2 will be further increased. Furthermore, when the power of S2 is above Brillouin threshold, S2 will make the third -order BS ( $\left.33, \omega_{\mathrm{S} 3}=\omega_{\mathrm{P}}-3 \Delta \omega_{\mathrm{B}}\right)$ to be generated on the same direction as $\mathrm{S} 1$. Hence, for $\mathrm{S} 1$ and $\mathrm{S} 3$ firstly take place the degenerate FWM process, which will produce the fifth-order Stokes signal $\left(\mathrm{S} 5, \omega_{\mathrm{S} 5}=2 \omega_{\mathrm{S} 3}-\omega_{\mathrm{S} 1}=\omega_{\mathrm{P}}-5 \Delta \omega_{\mathrm{B}}\right)$ and the first-order anti-Stokes signal (AS1, $\omega_{\mathrm{AS} 1}=2 \omega_{\mathrm{S} 1}-\omega_{\mathrm{S} 3}=\omega_{\mathrm{P}}+\Delta \omega_{\mathrm{B}}$ ) in anti-clockwise direction. These generated Stokes and anti-Stokes signals will interact and create the higher odd-order Stokes and anti-Stokes signals through multiple FWM processes. The illustration of SBS and multiple FWM processes within the fiber ring cavity observed from the counterclockwise direction is shown in Fig. 2a. Since odd-order Stokes and anti-Stokes circulate counterclockwise within the fiber ring cavity, they will output from the OUT1 port, and thanks to the RS of the BP signal and even-order Stokes and anti-Stokes signals, weak BP signal and even-order Stokes and anti-Stokes also circulate counterclockwise and output from the OUT1 port. Under strong BP conditions, the odd-order Stokes and anti-Stokes generated in the counterclockwise direction in the ring cavity get more gain than RS induced by the BP signal and even-order Stokes and anti-Stokes signals, which make the optical amplifier in the ring cavity being in deep saturation state, and these weak Rayleigh back light cannot be amplified. Therefore, the odd-order Stokes and anti-Stokes with double BFS are obtained from the OUT1 port. The illustration of SBS and multiple FWM processes within the fiber ring cavity observed from the clockwise direction are shown in Fig. 2b. Because BP signal and even -order Stokes and anti-Stokes circulate clockwise in the ring cavity, they will be output from the OUT2 port and due to the RS of odd-order Stokes and anti-Stokes signals, weak odd-order Stokes and anti-Stokes output from the OUT2 port. For the same reason, the odd-order Stokes and anti-Stokes with double BFS are obtained from the OUT2 port. Since odd- and even-order Stokes lines with double BFS have been obtained from the OUT1 and OUT2 port, respectively, switching from double BFS to single BFS can be achieved by reducing BP power. It can be explained as that, when BP power declines, power of Stokes decreases, and the optical amplifier gradually recovers from deep saturation state. Weak Rayleigh backscattering light begin to get the gain and be amplified, when the power of Rayleigh backscattering light increases to be close to or equal to that of the odd- and even-order Stokes. The MBEFL output with single BFS is achieved from the OUT1 and OUT2 port, respectively.

\section{Results and discussion}

Firstly, the amplified spontaneous emission (ASE) spectra of the EDFA are measured and shown in Fig. 3. In the employed EDFA is with a single-pump structure in order to distinguish the output direction of ASE, the direction the same as the transmission direction of the pump light is defined as forward, and the direction opposite to the transmission direction of the pump light is defined as backward. As can be seen from Fig. 3, 


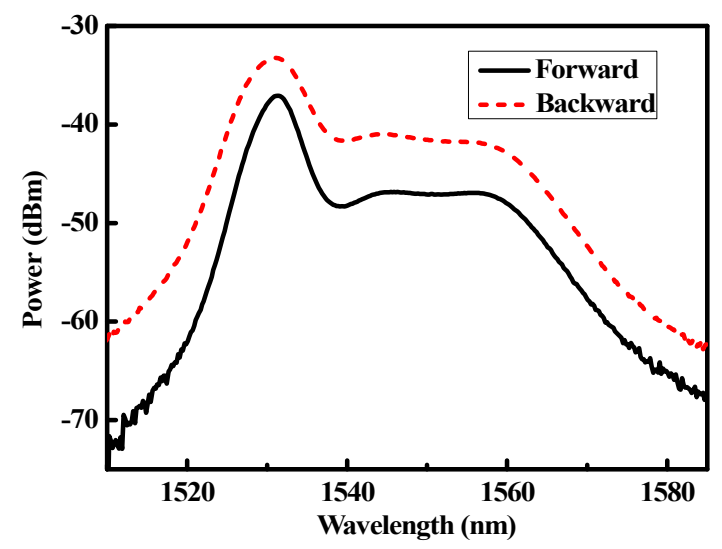

Fig. 3. The amplified spontaneous emission spectra of the erbium-doped fiber amplifier.

the forward gain of EDFA is less than the backward gain. This is because the employed EDF has extremely high doping concentration, which also means large attenuation coefficient. The forward output of the EDF is far from the pump light and has a larger attenuation, so the forward gain is relatively small. In order to obtain sufficient gain in both directions within the ring cavity, bidirectional pumping should be considered. However, because of the limitation of experimental conditions, only one pump source can be used in our experiment.

When the EDFA is connected into the ring cavity to be used and BP is shut down, the free running spectra of the fiber ring cavity output from the OUT1 and OUT2 port are shown in Fig. 4. The initial oscillation signal is generated from ASE of the EDFA in the fiber ring cavity. With the increase of pump power, some self-lasing cavity mode can be observed in the wavelength range of about $6 \mathrm{~nm}(1557-1563 \mathrm{~nm})$. Under the same pump conditions, the power of free-running spectrum output from OUT1 is slightly higher than that from OUT2. The reasons of that have been described above. In this

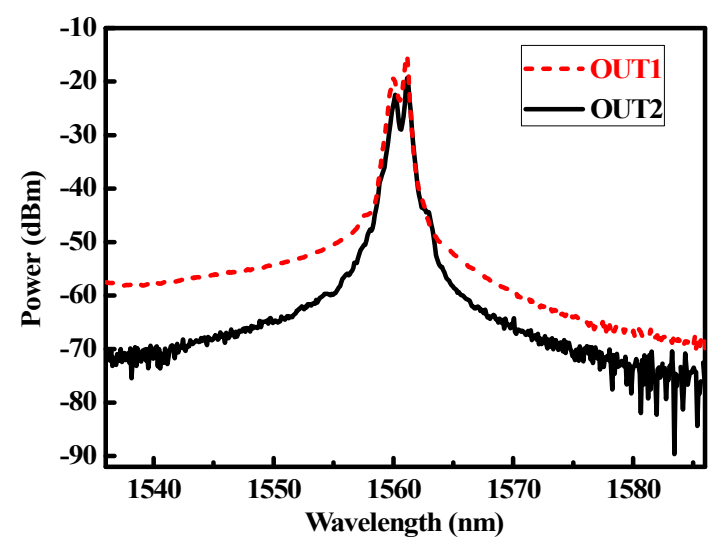

Fig. 4. Self-lasing spectra of the fiber ring cavity without Brillouin pump. 


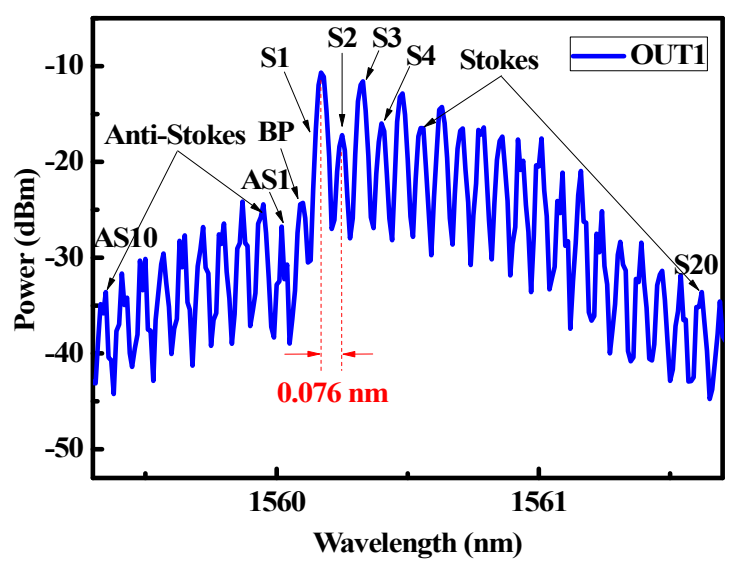

Fig. 5. Brillouin-Stokes with single Brillouin frequency spacing output from the OUT1 port.

case, all laser configurations operate under the free running condition, and unstable lasing modes are termed as stochastic modes. Since the cavity modes' oscillation and Brillouin based Stokes will induce gain competition in Brillouin-erbium fiber laser cavity, the wavelength of BP should locate within a small wavelength range near the cavity modes to suppress the cavity modes' competition [23]. Therefore, narrow free running bandwidth can limit wavelength tuning range of MBEFL.

Then, BP wavelength is set to $\sim 1560.094 \mathrm{~nm}$, which matches the peak value of output spectra of free-running laser, to trigger the generation of BS. When the BP power is $-1.44 \mathrm{dBm}$ and the power of the $980-\mathrm{nm}$ pump is $514 \mathrm{~mW}$, BS lines with single BFS of $0.076 \mathrm{~nm}$ are obtained from OUT1 and the number of generated Stokes lines exceeds 20, as shown in Fig. 5. Because the most energy of the BP and even-order Stokes circulates on the clockwise direction in the ring cavity, the BP and even-order Stokes signal are also scattered in the counterclockwise direction along the fiber via the Rayleigh feedback effect. Since there is no optical ISO in the ring cavity, the power of bidirectional circular light fields in the cavity can be amplified in the EDFA. Because the BP power is relatively low, the power of the odd-order Stokes generated in the counterclockwise direction is not too high, as they pass through the EDFA and is slightly amplified. But, BP and even-order Stokes-induced Rayleigh backscattering light power are relatively low and their power is amplified more. When the power of the even-order Stokes -induced Rayleigh backscattering light increases to be close to or equal to that of the odd-order Stokes, the gain contention results in the Stokes sequence with single BFS is obtained.

By gradually increasing the BP power, the odd-order Stokes lines with double BFS are obtained from the OUT1 port of the MBEFL, as shown in Fig. 6. When the BP power reaches $10.42 \mathrm{dBm}$, the $980 \mathrm{~nm}$ pump power is $662 \mathrm{~mW}$. It can be seen that within the spectrum range from 1559.11 to $1562.14 \mathrm{~nm}$, there are twenty Stokes lines, including odd-order Stokes components from the first order to the twenty-seventh order, and six odd-order anti-Stocks components. The frequency spacing between two adjacent 


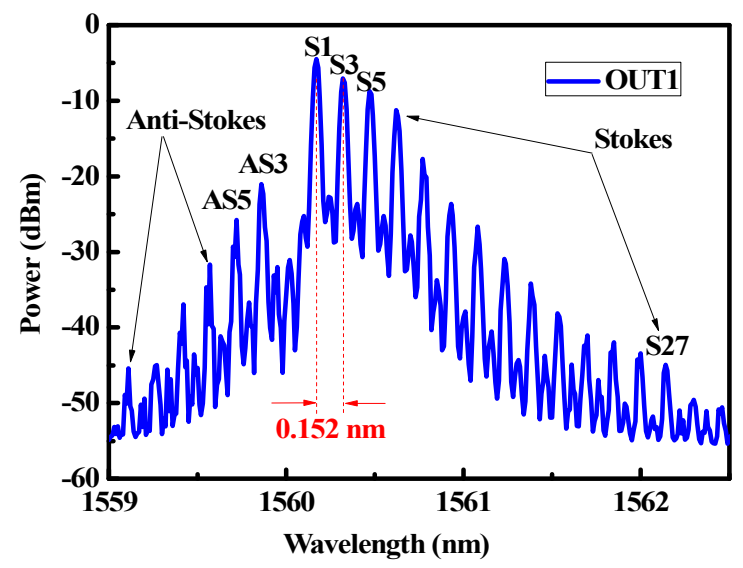

Fig. 6. Odd-order Brillouin-Stokes with double Brillouin frequency spacing output from the OUT1 port.

wavelengths is around $0.152 \mathrm{~nm}$, which is double of the basic SBS frequency shifting. The BP and even-order Stokes-induced Rayleigh backscattering light is suppressed and the odd-order Stokes signals are amplified to the maximum extent possible by adjusting polarization state of PCs, since both FWM and SBS are polarization-dependent.

Since the BP power may be a key system parameter that affects the laser output, the effect of BP power on the laser output is investigated and the results are reported in Fig. 7. The power of the $980 \mathrm{~nm}$ pump is kept on $662 \mathrm{~mW}$. When the BP power is very low and equals $-16.25 \mathrm{dBm}$, only ASE noise outputs from the laser. With increas-

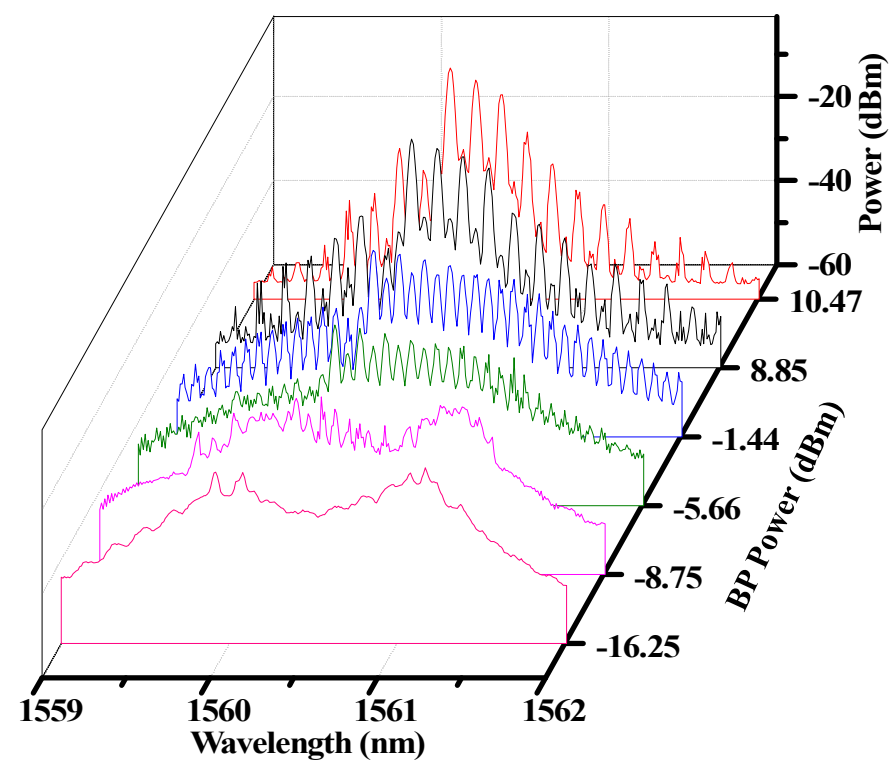

Fig. 7. Output spectra of the multi-wavelength Brillouin-erbium fiber laser at the different Brillouin pump power. 
ing of BP power, the Brillouin comb lines begin to appear and gradually increase. When the BP power is $-1.44 \mathrm{dBm}$, Stokes lines with single BFS are obtained from the OUT1 port. When BP power increases to $10.47 \mathrm{dBm}$, even-order Stokes lines are suppressed, and odd-order Stokes lines with double BFS are obtained from the OUT1 port. Thus, it can be seen that the BP power plays an extremely important role in realizing Stokes generation with switchable frequency spacing.

The influence of EDFA pump power on Stokes generation from MBEFL is also investigated. The wavelength of BP light is set at $1560.094 \mathrm{~nm}$, and BP power is fixed at $10.42 \mathrm{dBm}$. Only by adjusting EDFA pump power, different output spectra of MBEFL are obtained from the OUT1 port, as shown in Fig. 8. When EDFA pump power is $50 \mathrm{~mW}$, only BP-induced Rayleigh backscattering light is observed. When EDFA pump power increases to $134 \mathrm{~mW}$, the 1st order Stokes lasing based on SBS is initiated by BP, and only two Stokes peaks are observed, namely BP-induced Rayleigh backscattering light and 1st order Stokes lasing, respectively. When EDFA pump power further increases to $248 \mathrm{~mW}$, the main peak accompanied by two small side peaks are observed, referring to the 1st BS, BP and 2nd BS-induced Rayleigh backscattering light, respectively. The measurement for stability of the 1st Stokes output spectrum being taken over more than 20 minutes reveals that there are almost no observable fluctuations in the peak power and central wavelength over the measured period, which indicates that 1 st order Stokes lasing is very stable. When EDFA pump power is equal to $262 \mathrm{~mW}$, in addition to the stable 1 st Stokes lasing output, the 3rd stimulated BS lasing amplified is newly generated. When EDFA pump power is $362 \mathrm{~mW}$, in addition to the stable 1 st and 3rd Stokes lasing outputs, the 5th stimulated BS and amplified 1st anti-BS are

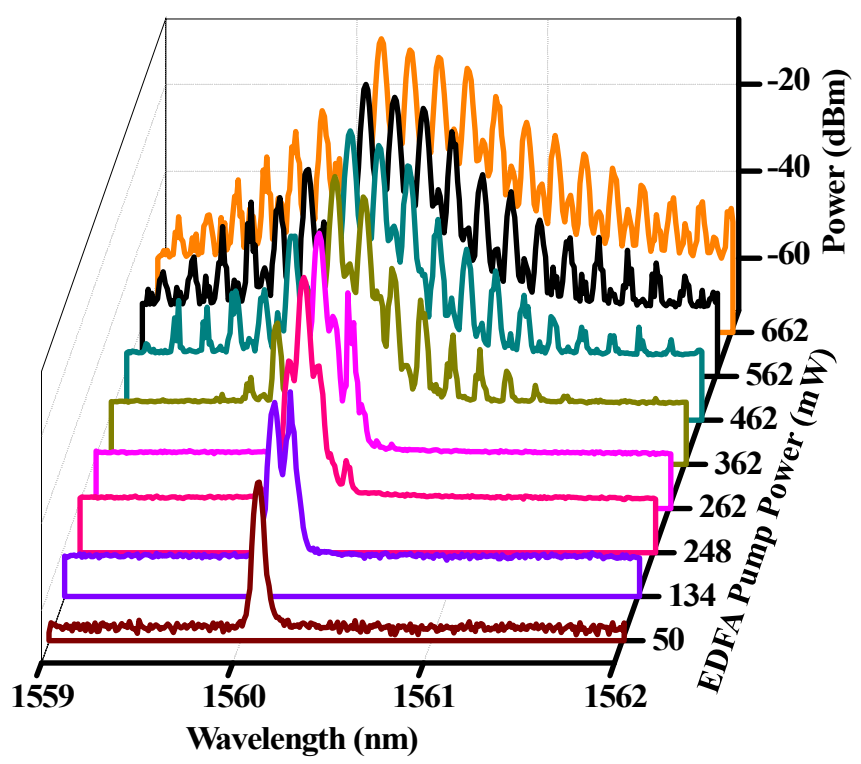

Fig. 8. Output spectra of the multi-wavelength Brillouin-erbium fiber laser on the different erbium-doped fiber amplifier pump power. 
newly initiated. With increasing of EDFA pump power, the lasing wavelength number of MBEFL output increases. From another perspective, the proposed MBEFL can realize switchable odd-order Stokes generation, with an increasing wavelength number in turn, only by simply adjusting EDFA pump power. When BP power is fixing at $-1.44 \mathrm{dBm}$, by adjusting EDFA pump power, similar phenomena can be observed. It means that the proposed MBEFL also can realize switchable Stokes generation with single BFS, with an increasing wavelength number in turn, only by simply adjusting EDFA pump power.

The even-order Stokes lines with double BFS from the OUT2 port of the MBEFL are shown in Fig. 9, when the BP wavelength is $1560.094 \mathrm{~nm}$ and EDFA pump power is $691 \mathrm{~mW}$. It can be seen that thirteen even-order Stokes and anti-Stokes components within the spectrum range of $1558.35-1561.31 \mathrm{~nm}$ are generated. The frequency spacing between two adjacent wavelengths is $0.152 \mathrm{~nm}$. By reducing the BP power, the even-order Stokes generation with double BFS is switched to the Stokes generation

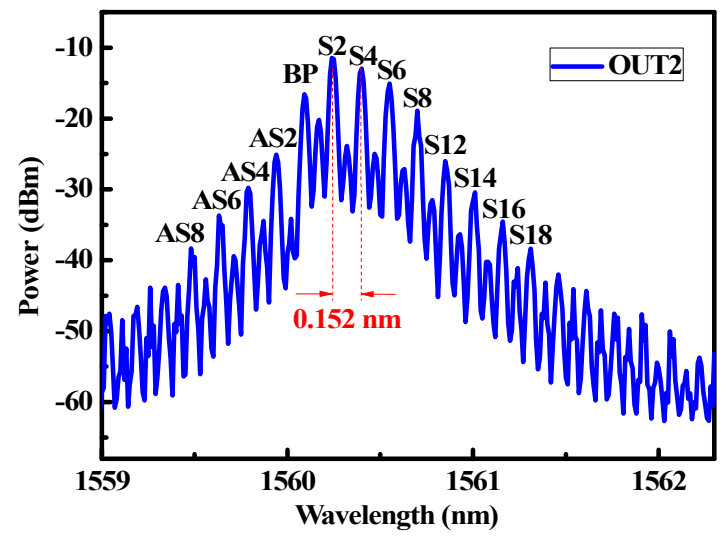

Fig. 9. The even-order Stokes with double Brillouin frequency spacing output from the OUT2 port.

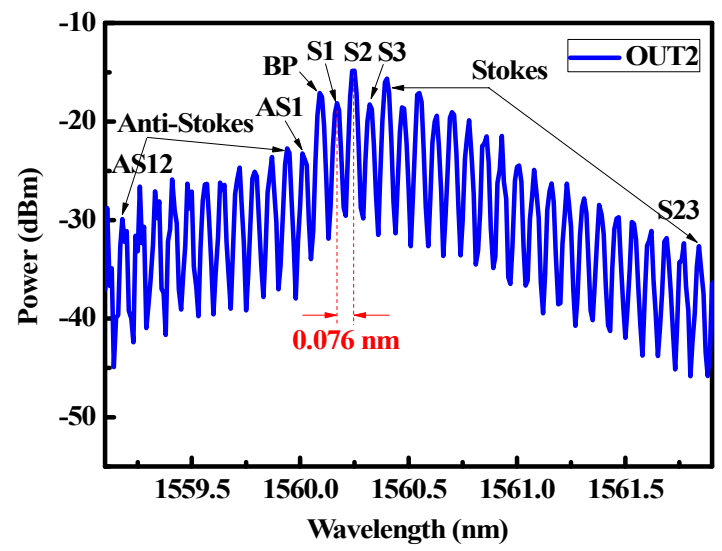

Fig. 10. Stokes with single Brillouin frequency spacing output from the OUT2 port. 
with single BFS at the OUT2 port of the MBEFL. When the BP power is $3.2 \mathrm{dBm}$, the Stokes lines with single BFS are generated, as shown in Fig. 10. The frequency spacing between two adjacent wavelengths is $0.076 \mathrm{~nm}$.

Since stability is one of the most important system properties for MBEFL, the repeated spectra of MBEFL output with single BFS from the OUT2 port, within 60 minutes with the time interval of 10 minutes, are recorded and evaluated, as shown in Fig. 11, when the BP wavelength is $1560.094 \mathrm{~nm}$ and the pump power of EDFA is

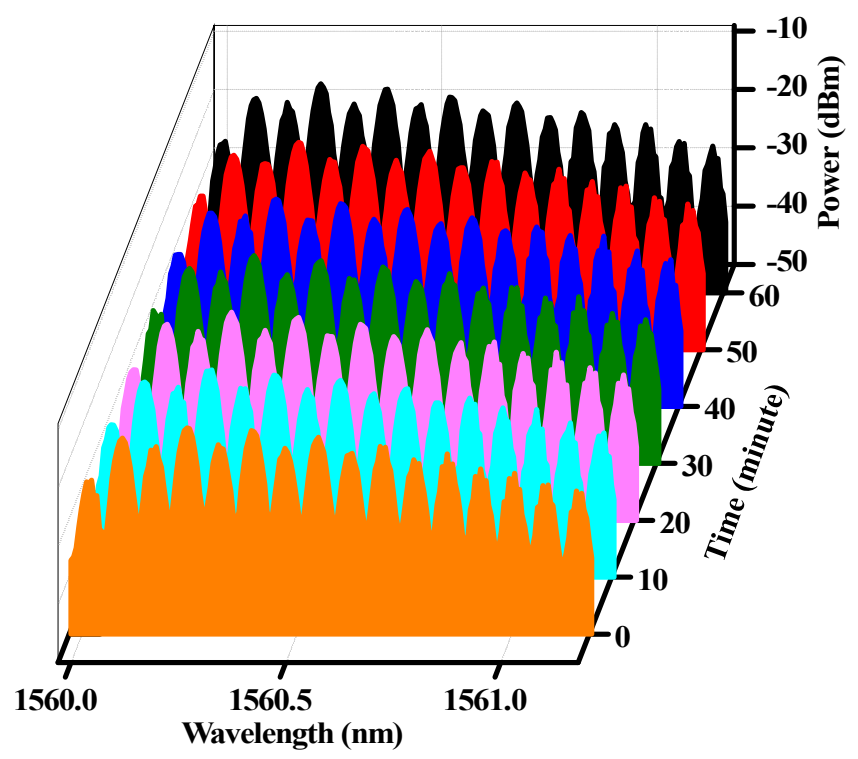

Fig. 11. Repeated scanning spectra over 60 minutes with the interval of 10 minutes.

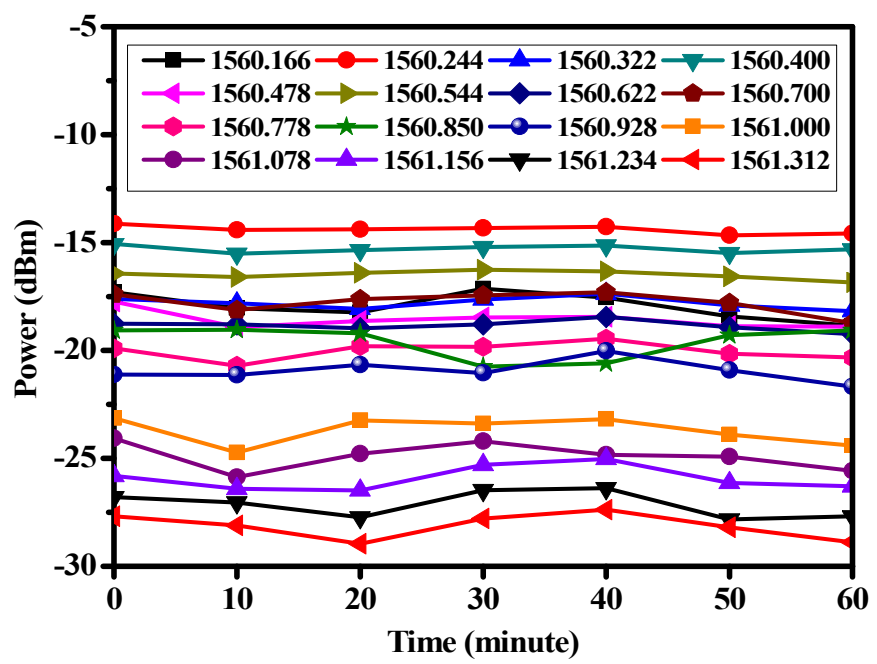

Fig. 12. The peak power fluctuations for the channels from the 1 st to 16 th. 
$623 \mathrm{~mW}$. As you seen, Stokes lines from the 1st to 16th are stable with time in 60-minute duration. To further highlight stability of the laser, the peak power fluctuation for the channel from the 1st to 16 th are plotted in Fig. 12. The peak power fluctuations recorded are 1.71, 0.54, 0.80, 0.45, 1.17, 0.58, 0.81, 1.44, 1.26, 1.72, 1.64, 1.59, 1.80, $1.46,1.45$, and 1.58 , respectively. These low fluctuations of less than $1.80 \mathrm{dBm}$ within 60 minutes demonstrate the stability of the proposed MBEFL.

The wavelength tunability of MBEFL can provide flexibility of system applications; so, the wavelength tunability of the proposed MBEFL is also investigated and reported in Fig. 13. The BP power is fixed at $3.2 \mathrm{dBm}$ and EDFA pump power is kept on $623 \mathrm{~mW}$. When the BP wavelength is tuned within a range of about $7 \mathrm{~nm}$ from 1557.075 to $1564.073 \mathrm{~nm}$, stable and clear MBEFL output can be obtained from the OUT2 port. Wavelength tuning range of the proposed MBEFL is relatively narrow, which is limited by the wavelength range of self-lasing cavity mode. Because the cavity modes' oscillation and Brillouin based Stokes induce the gain competition in Brillouin -erbium fiber laser cavity, the wavelength of BP is selected to near the wavelength of the cavity modes to suppress the cavity modes' competition. The limited wavelength tuning range, the limited number of lasing modes, and the low amplitude equalization degree of the lasing modes may be related to the SBS gain and stimulated radiation gain. Sufficient SBS gain is a necessary condition for achieving broadband lasing, which requires BP source to provide sufficient BP power. To realize the bidirectional oscillation output, BP light should be injected into the ring cavity via OC. However, the use of $\mathrm{OC}$ in the ring cavity will inevitably lead to optical field diversion and energy loss. In order to compensate for the energy loss of the light field, the amplifier is connected to the ring cavity, which also will lead to the cavity mode competition and narrow gain bandwidth. Therefore, the SBS gain and the stimulated radiation gain should be coordinated in the fiber ring cavity. The SBS gain should be dominant, while the stimulated radiation gain is only used to compensate for the energy loss of the optical

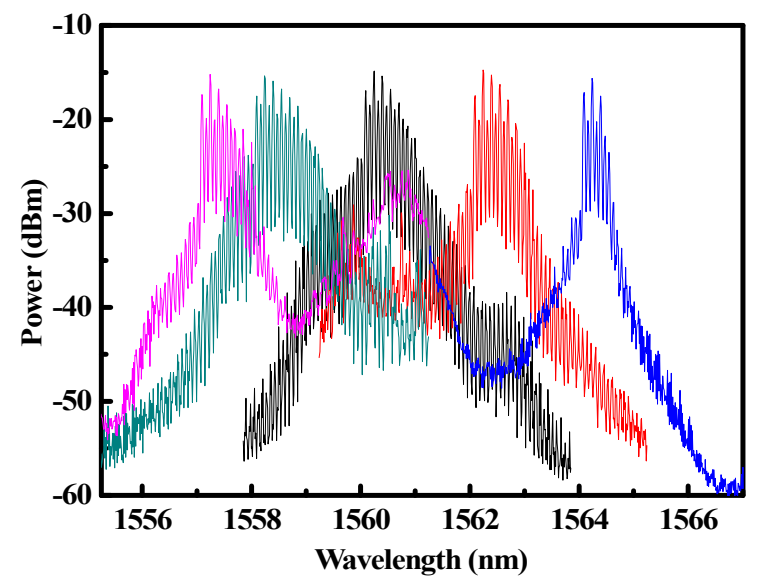

Fig. 13. Output spectra of the multi-wavelength Brillouin-erbium fiber laser with single Brillouin frequency spacing for different Brillouin pump wavelengths. 
field. In addition, in order to avoid the conflict between SBS gain and stimulated radiation gain, other forms of cavity structures can also be considered, such as half-open cavity structures.

\section{Conclusion}

In summary, a MBEFL with a switchable frequency space has been experimentally demonstrated, switching between single and double BFS is conveniently achieved only by adjusting BP power. Moreover, under the certain BP power conditions, the proposed MBEFL also can realize switchable odd- or even-order Stokes generation with double BFS and Stokes generation with single BFS, with an increasing wavelength number in turn, only by simply adjusting EDFA pump power. The proposed MBEFL may find some applications in various fields such as high-capacity optical communications, low -noise tunable microwave or $\mathrm{THz}$ wave generation and spectroscopic studies.

Acknowledgment - This work was supported by the Fundamental Research Funds for the Central Universities of China (SWU020001), National Natural Science Foundation of China (61575034), and Recruitment program of global experts (WQ20165000357).

\section{References}

[1] Bellemare A., Karásek M., Rochette M., LRochelle S., TÊtu M., Room temperature multifrequency erbium-doped fiber lasers anchored on the ITU frequency grid, Journal of Lightwave Technology 18(6), 2000, pp. 825-831, DOI: 10.1109/50.848393.

[2] Xu R., Zhang X., Multiwavelength Brillouin-erbium fiber laser temperature sensor with tunable and high sensitivity, IEEE Photonics Journal 7(3), 2015, article 1501708, DOI: 10.1109/JPHOT.2015. 2422132.

[3] Wang Z., Wang T., Jia Q., Ma W., Su Q., Zhang P., Triple Brillouin frequency spacing multiwavelength fiber laser with double Brillouin cavities and its application in microwave signal generation, Applied Optics 56(26), 2017, pp. 7419-7426, DOI: 10.1364/AO.56.007419.

[4] Camarillo-Avilés A., Jauregui-Vazquez D., Estudillo-Ayala J.M., Hernández-Escobar E., Sierra-Hernández J.M., Pottiez O., Durán-SÁnchez M., Ibarra-Escamilla B., Bello-Jiménez M., Stable multi-wavelength thulium-doped all-fiber laser incorporating a multi-cavity Fabry-Perot filter, IEEE Photonics Journal 11(6), 2019, article 7105307, DOI: 10.1109/JPHOT.2019.2949500.

[5] Zhang L., Yan F., Feng T., Guo Y., Qin Q., Zhou H., Suo Y., Switchable multi-wavelength thulium -doped fiber laser employing a polarization-maintaining sampled fiber Bragg grating, IEEE Access 7, 2019, pp. 155437-155445, DOI: 10.1109/ACCESS.2019.2944168.

[6] Luo A.P., Luo Z.C., Xu W.C., Tunable and switchable multiwavelength erbium-doped fiber ring laser based on a modified dual-pass Mach-Zehnder interferometer, Optics Letters 34(14), 2009, pp. 2135 -2137, DOI: 10.1364/OL.34.002135.

[7] Jasim A.A., Dernaika M., Harun S. W., Ahmad H., A switchable figure eight erbium-doped fiber laser based on inter-modal beating by means of non-adiabatic microfiber, Journal of Lightwave Technology 33(2), 2015, pp. 528-534, DOI: 10.1109/j1t.2015.2390654.

[8] Ummy M.A., Madamopoulos N., Joyo A., Kouar M., Dorsinville R., Tunable multi-wavelength SOA based linear cavity dual-output port fiber laser using Lyot-Sagnac loop mirror, Optics Express 19(4), 2011, pp. 3202-3211, DOI: 10.1364/OE.19.003202. 
[9] Norcia S., Tonda-Goldstein S., Dolfi D., Huignard J.P., Frey R., Efficient single-mode Brillouin fiber laser for low-noise optical carrier reduction of microwave signals, Optics Letters 28(20), 2003, pp. 1888-1890, DOI: $10.1364 /$ OL.28.001888.

[10] Li Q., Jia Z.X., Weng H.Z., Li Z.R., YAng Y.D., Xiao J.L., Chen S.W., Huang Y.Z., Qin W.P., Qin G.S., Broadband multi-wavelength Brillouin lasers with an operating wavelength range of 1500-1600 nm generated by four-wave mixing in a dual wavelength Brillouin fiber laser cavity, Laser Physics Letters 15(5), 2018, article 055103, DOI: 10.1088/1612-202X/aaafb9.

[11] Kuo H.L., Lin W.P., Double-Brillouin-frequency spaced multiwavelength generation in one loop structure, Optics Letters 39(15), 2014, pp. 4565-4567, DOI: 10.1364/OL.39.004565.

[12] Mamdoohi G., Sarmani A.R., BaKar M.H.A., Mahdi M.A., Switchable multiwavelength Brillouin -Raman fiber laser utilizing an enhanced nonlinear amplifying fiber loop design, IEEE Photonics Journal 10(2), 2018, article 7201011, DOI: 10.1109/JPHOT.2018.2809557.

[13] QIAN L., Fen D., XIE H., Sun J., A novel tunable multi-wavelength Brillouin fiber laser with switchable frequency spacing, Optics Communications 340, 2015, pp. 74-79, DOI: 10.1016/j.optcom. 2014.11.091.

[14] Wang X., Yang Y., Liu M., Yuan Y., Sun Y., Gu Y., Yao Y., Frequency spacing switchable multiwavelength Brillouin erbium fiber laser utilizing cascaded Brillouin gain fibers, Applied Optics 55(23), 2016, pp. 6475-6479, DOI: 10.1364/AO.55.006475.

[15] Al-Mansoori M.H., Abd-Rahman M.K., Adikan F.R.M., Mahdi M.A., Widely tunable linear cavity multiwavelength Brillouin-erbium fiber lasers, Optics Express 13(9), 2005, pp. 3471-3476, DOI: 10.1364/OPEX.13.003471.

[16] Samsuri N.Md., Zamzuri A.K., Al-Mansoori M.H., Ahmad A., Mahdi M.A., Brillouin-erbium fiber laser with enhanced feedback coupling using common erbium gain section, Optics Express 16(21), 2008, pp. 16475-16480, DOI: 10.1364/OE.16.016475.

[17] Al-Alimi A.W., Sarmani A.R., Al-Mansoori M.H., Lakshminarayana G., Mahdi M.A., Flat amplitude and wide multiwavelength Brillouin/erbium fiber laser based on Fresnel reflection in a micro -air cavity design, Optics Express 26(3), 2018, pp. 3124-3137, DOI: 10.1364/OE.26.003124.

[18] Li H., Hu F., Li C., Tian Y., Huang C., Zhang J., Xu S., Generation of switchable multiwavelength solitons with wide wavelength spacing at $2 \mu \mathrm{m}$, Optics Letters 44(10), 2019, pp. 2442-2445, DOI: 10.1364/OL.44.002442.

[19] Hu K., Kabakova I.V., Lefrancois S., Hudson D.D., He S., Eggleton B.J., Hybrid Brillouin/thulium multiwavelength fiber laser with switchable single-and double-Brillouin-frequency spacing, Optics Express 22(26), 2014, pp. 31884-31892, DOI: 10.1364/OE.22.031884.

[20] Wang X., Zhou P., Wang X., XIAo H., Si L., Multiwavelength Brillouin-thulium fiber laser, IEEE Photonics Journal 6(1), 2014, article 1500507, DOI: 10.1109/JPHOT.2013.2295471.

[21] WANG Z., Wu H., FAn M., Li Y., Gong Y., RaO Y., Broadband flat-amplitude multiwavelength Brillouin-Raman fiber laser with spectral reshaping by Rayleigh scattering, Optics Express 21(24), 2013, pp. 29358-29363, DOI: 10.1364/OE.21.029358.

[22] Shen Y., Zhang X., Chen K., Stimulated Brillouin scattering for efficient improvement of radio-over -fiber systems, Optical Engineering 44(10), 2005, article 105003, DOI: 10.1117/1.2087628.

[23] Dong B., Zhou D.P., Wei L., Tunable multiwavelength Brillouin-erbium fiber laser by controlling self-lasing cavity modes' oscillation, Optical Fiber Technology 16(1), 2010, pp. 17-19, DOI: 10.1016/ j.yofte.2009.09.008. 